\title{
Letter
}

\section{Permeability of skin to silver nanoparticles after epidermal skin barrier disruption in rats}

\author{
Makiko Kuwagata', Fumiaki Kumagai'1, Yoshiaki Saito', Kazuma Higashisaka², \\ Yasuo Yoshioka2,3 and Yasuo Tsutsumi² \\ ${ }^{1}$ Hatano Research Institute Food and Drug Safety Center, 729-5 Ochiai, Hadano, Kanagawa 257-8523, Japan \\ ${ }^{2}$ Laboratory of Toxicology and Safety Science Graduate School of Pharmaceutical Science Osaka University, \\ 1-6 Yamadaoka, Suita, Osaka, 565-0871, Japan \\ ${ }^{3}$ Vaccine Creation Project, BIKEN Innovative Vaccine Research Alliance Laboratories, Research Institute for \\ Microbial Diseases, Osaka University, 1-6 Yamadaoka, Suita, Osaka, 565-0871, Japan
}

(Received April 24, 2017; Accepted May 2, 2017)

\begin{abstract}
To analyze the permeability of rat skin to silver nanoparticles, the dorsal skin of Sprague-Dawley rats was exposed to $5 \mathrm{~nm} \mathrm{Ag}$ nanoparticles or silver nitrate ( $\mathrm{Ag}^{+}$ions) percutaneously for $24 \mathrm{hr}$ after disruption of the epidermal barrier by tape stripping (TS) or acetone wiping (AC). Systemic toxicity was examined hematologically and histopathologically, and by assessing blood biochemistry. Although parakeratosis, decrease in keratohyaline granule, and thickening in the epidermis occurred following exposure to both $5 \mathrm{~nm} \mathrm{Ag}$ nanoparticles and $\mathrm{Ag}^{+}$ions after TS or AC, no Ag-specific changes were observed. Inductively coupled plasma mass spectrometry (ICP-MS) showed silver in the skin of rats exposed to both $5 \mathrm{~nm} \mathrm{Ag}$ nanoparticles and $\mathrm{Ag}^{+}$ions after TS or AC. Silver was only detected in the liver of rats exposed to $\mathrm{Ag}^{+}$ions after TS, but not exposed to $5 \mathrm{~nm} \mathrm{Ag}$ nanoparticles after TS or AC. No abnormal histopathological changes in the liver were observed in all rats. In the blood, silver was below detectable levels in all rats and had no adverse effects on hematology or blood biochemistry. These results indicate that silver ions released from $5 \mathrm{~nm} \mathrm{Ag}$ nanoparticles can percutaneously infiltrate the body only when the skin barrier is disrupted, but does not induce any acute toxicity.
\end{abstract}

Key words: Silver nanoparticle, Transcutaneous exposure, Rat, Epidermal barrier disruption

\section{INTRODUCTION}

Silver nanoparticles are one of the most frequently used nanomaterials in consumer products because of their antimicrobial properties and relatively low toxicity in humans (Ahamed et al., 2010; Avalos et al., 2014; Brandt et al., 2012; Chen and Schluesener, 2008). The antibacterial activity of silver nanoparticles increases with decreasing particle size, an inverse relationship associated with the increased surface area-to-mass ratio of small particles (Ahamed et al., 2010). The degree of absorption is mainly governed by nanoparticle size, surface charge, hydrophobicity, and the presence or absence of surface ligands (Kokura et al., 2010; Loeschner et al., 2011).

Silver nanoparticles have toxic effects in vitro on animal and human cell lines, including the induction of oxidative stress, DNA damage and apoptosis (Avalos et al., 2014; Kim and Ryu, 2013). In in vivo studies, administra- tion of intact silver nanoparticles to animals orally (Juling et al., 2016; Kim et al., 2008; Kim et al., 2010; Loeschner et al., 2011; Rigo et al., 2013), by inhalation (Kim et al., 2008; Loeschner et al., 2011), intravenously (De Jong et al., 2013; Lankveld et al., 2010), subcutaneously (Tang et al., 2009), and transdermally (Korani et al., 2011; Samberg et al., 2010) have been shown to result in the translocation and deposition of these nanoparticles into various organs, as well as several adverse effects.

Assessment of the toxicity of silver nanoparticles, especially those contained in consumer products, should include the effects of dermal exposure. The skin is the largest organ of the body, accounting for up to $10 \%$ of body mass, as well as being a highly effective interface between an organism and the external environment, including nanoparticles (Gujjar and Banga, 2014; Labouta and Schneider, 2013; Ostrowski et al., 2014; Patzelt and Lademann, 2013). Penetration is thought to be affected by various fac-

Correspondence: Makiko Kuwagata (E-mail: kuwagata.m@fdsc.or.jp) 
tors, especially by dermal structure and integrity (Brouwer et al., 2016; Crosera et al., 2009). In particular, skin diseases involving barrier alternations, which are common in humans, may alter the penetration of nanoparticles (Avalos et al., 2014; Brouwer et al., 2016; Larese Filon et al., 2015; Rigo et al., 2013).

Silver nanoparticles are reemerging as a viable topical treatment option for infections encountered in burns, open wounds and chronic ulcers, making permeability of skin to these nanoparticles important (Avalos et al., 2014; Rigo et al., 2013). Percutaneous absorption of silver nanoparticles is still a matter of investigation by researchers because of different view (Ahamed et al., 2010; Bianco et al., 2015a; Munger et al., 2014; Samberg et al., 2010; Stefaniak et al., 2014). Thus, accumulation of reliable data of animal study to examine the penetration of nanoparticles under damaged skin condition should be important for risk assessment.

This study examined whether very small-sized silver nanoparticles can permeate rat skin following barrier disruption by tape stripping or acetone wiping, as well as whether permeating silver nanoparticles induce acute toxicity in these animals.

\section{MATERIALS AND METHODS}

All animal care and experimental procedures were approved by the Institutional Animal Care and Use Committee of the Food and Drug Safety Center, Hatano Research Institute.

\section{Silver nanoparticles and sample preparation}

Solutions of citrate-coated silver nanoparticles $5 \mathrm{~nm}$ in diamter ( $5 \mathrm{~nm}$ Citrate Biopure ${ }^{\mathrm{TM}}$ silver, $5 \mathrm{~nm} \mathrm{Ag}$, Lot number MGM2206, $1 \mathrm{mg} / \mathrm{mL}$ ) were purchase from nanoComposix Inc. (San Diego, CA, USA). These nanoparticles were maintained in stable suspension, to avoid their aggregation. The measured mean diameter of the silver nanoparticles was $4.6 \pm 0.8 \mathrm{~nm}$ and their mean concentration was $1.09 \mathrm{mg} / \mathrm{mL}$ in $0.2 \mathrm{mM}$ citric acid (Fig. 1), with $<5 \%$ being ionized.

As a positive control, a solution of $0.1 \mathrm{M}$ silver nitrate ( $\mathrm{AgNO}_{3}, \mathrm{Ag}^{+}$ions, Wako Chemical Industries Ltd.) was diluted to $1 \mathrm{mg} / \mathrm{mL}$ in $0.2 \mathrm{mM}$ citric acid. The vehicle control consisted of $0.2 \mathrm{mM}$ citric acid (Wako, Osaka, Japan). The dosing volume was $4 \mathrm{~mL} / \mathrm{kg}$.

\section{Animals and study protocol}

Six-week-old male Sprague-Dawley [Crl:CD (SD), $\mathrm{SPF}$ ] rats were purchased from Japan Charles River Laboratory (Atsugi, Japan) and housed in a room at the

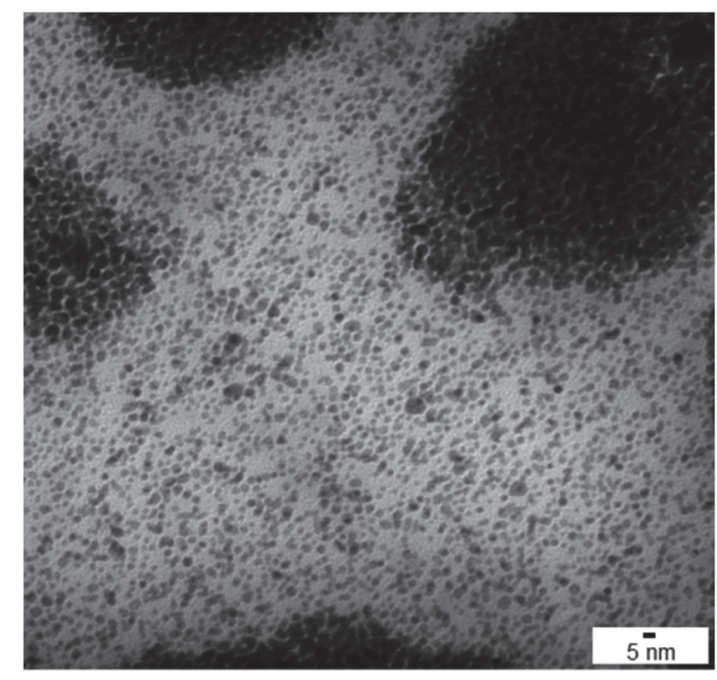

Fig. 1. Transmission electron micrograph of $5 \mathrm{~nm}$ silver nanoparticles, showing good spreading.

Animal Facility of Hatano Research Institute. Temperature and relative humidity were controlled, with a 12-12 hr light-dark cycle (lights on at 07:00). The animals were fed standard rat chow (CE-2, CLEA, Japan) and tap water (Hadano City municipal water) ad libitum and allowed to acclimate for one week after arrival. At age 7 weeks, the rats were randomly divided into seven groups of 4-11 rats per group based on body weight. The hair on the backs of these rats was shaved with an electrical shaver (Thrive ${ }^{\circledR}$, Osaka, Japan) and hair clipper (Panasonic ${ }^{\circledR}$, Osaka, Japan). The dorsal skin barrier was subsequently disrupted by tape stripping (cellophane tape, NICHIBAN, Tokyo, Japan) 20 times (TS), or by wiping with acetone (Wako) 10 times per day using a small cotton stick for 4 continuous days (AC).

Rats were subsequently administered $5 \mathrm{~nm}$ Ag nanoparticles, $0.1 \mathrm{M} \mathrm{AgNO}_{3}\left(\mathrm{Ag}^{+}\right.$ions $)$, or $0.2 \mathrm{mM}$ citric acid (vehicle control) percutaneously for $24 \mathrm{hr}$. A patch containing each agent $(3 \times 3 \mathrm{~cm})$ was applied to the skin and covered with non-absorbing film $\left(6 \times 7 \mathrm{~cm}\right.$, Tegaderm ${ }^{\mathrm{TM}}$ HP, 3M, Minneapolis, USA), size of $3 \times 30 \mathrm{~cm}$ plastic wrap with a self-adhering foam pad (Reston $\left.{ }^{\mathrm{TM}}, 3 \mathrm{M}\right)$, and three cutting $5 \times 30 \mathrm{~cm}$ stretch bandages (Silky tex No.5, Alcare Co. Ltd., Tokyo, Japan).

After 24 hours (day 2), the bandages were removed. Rats pretreated with TS were sacrificed on days 2, 3, and 5 by injection with sodium pentobarbital. On day 4 , rats in this group were fasted overnight, and blood samples for hematological and biochemical analyses were collected at autopsy on day 5. Organ weights were measured, tis- 
Nano-silver permeability of rat skin

sue samples obtained, and histopathological examinations performed. Rats pretreated for 4 days with AC, following which $5 \mathrm{~nm} \mathrm{Ag} \mathrm{nanoparticles,} \mathrm{Ag}^{+}$ions, or vehicle was applied, were autopsied $24 \mathrm{hr}$ after the exposure (day 2). Organ weights were measured, tissue samples obtained, and histopathological examinations performed. The study design, showing randomization of rats to pretreatment with TS or AC and treatment with $5 \mathrm{~nm} \mathrm{Ag} \mathrm{nanoparticles,}$ $\mathrm{Ag}^{+}$ions, or vehicles, is shown in Fig. 2.

\section{Determination of $\mathrm{Ag}$ in the tissue}

The concentrations of Ag in the skin, liver, and blood were analyzed by inductively coupled plasma mass spectrometry (ICP-MS) on days 2, 3, and 5 in rats pretreated with TS, and on the day 2 in rats pretreated with AC. The limit of detection was less than $0.05 \mathrm{ppm}$. Animals not pretreated with TS or AC were assessed similarly. At each point, two animals were selected randomly for analysis.

\section{Statistical analysis}

Hematologic and biochemical data, as well as organ weights, were summarized as group means and standard deviations. The level of statistical significance was set at $\mathrm{p}<0.05$.

\section{RESULTS}

No animal died and no abnormal clinical signs were observed during the study period.

As a preliminary data, changes in the epidermis just after TS or AC were examined, with histopathological changes assessed by hematoxylin-eosin (HE) staining of paraffin-embedded sections (Fig. 3). Macroscopic examination showed scattered reddish spots under skin after TS or AC treatment, with more spots observed after TS than after AC treatment (Fig. 3A, 3C, 3E). Histopathological examination showed that TS treatment resulted in stripping of most of stratum corneum and the degeneration of epidermal keratinocyte. AC treatment resulted in parakeratosis with slight thickening of the epidermal layer and the stratum corneum was thin, with deficient cornification and a decrease in the number of keratohyaline granules. These findings indicated that TS treatment induced more severe injury than AC treatment.

\begin{tabular}{|c|c|c|c|c|c|c|}
\hline & & Day 1 & Day 2 & Day 3 & Day 4 & Day 5 \\
\hline & & $\begin{array}{c}\hat{\uparrow} \\
\mathrm{TP}\end{array}$ & & & $\begin{array}{c}\uparrow \\
\text { Fasting }\end{array}$ & \\
\hline & & \multicolumn{2}{|c|}{ 24hr exposure } & & & \\
\hline \multicolumn{7}{|c|}{ Number of animals } \\
\hline Control & TS+ & [11] & 4 & 2 & & 5 \\
\hline \multirow[t]{2}{*}{$5 \mathrm{~nm} \mathrm{Ag}$} & TS+ & {$[11]$} & 4 & 2 & & 5 \\
\hline & TS- & [ 7 ] & 2 & & & 5 \\
\hline $\mathrm{AgNO}_{3}$ & TS+ & [11] & 4 & 2 & & 5 \\
\hline \multicolumn{3}{|c|}{$\begin{array}{l}\text { Autopsy (ICP-MS analysis) } \\
\text { Systemic toxicity evaluation }\end{array}$} & 0 & 0 & & $\begin{array}{l}0 \\
0\end{array}$ \\
\hline
\end{tabular}

(B) AC group

\begin{tabular}{|c|c|c|c|c|c|}
\hline & $\mathrm{T} 1$ & $\mathrm{~T} 2$ & $\mathrm{~T} 3$ & T4 (Day 1) & Day 2 \\
\hline & $\mathrm{AC}$ & $\mathrm{AC}$ & $\mathrm{AC}$ & $\mathrm{AC}$ & \\
\hline & & & & & \\
\hline Number of animals & & & & & \\
\hline Control $\mathrm{AC}+$ & [ 4 ] & & & & 4 \\
\hline $5 \mathrm{~nm} \mathrm{Ag} \quad \mathrm{AC}+$ & [ 4 ] & & & & 4 \\
\hline $\mathrm{AgNO}_{3} \quad \mathrm{AC}+$ & [ 4 ] & & & & 4 \\
\hline Autopsy (ICP-MS & & & & & 0 \\
\hline
\end{tabular}

Fig. 2. Study design, showing pre-treatment of groups of rats with TS, AC or no treatment; followed by treatment with $5 \mathrm{~nm} \mathrm{Ag}$ nanoparticles, $\mathrm{Ag}^{+}$ions, or vehicle. 

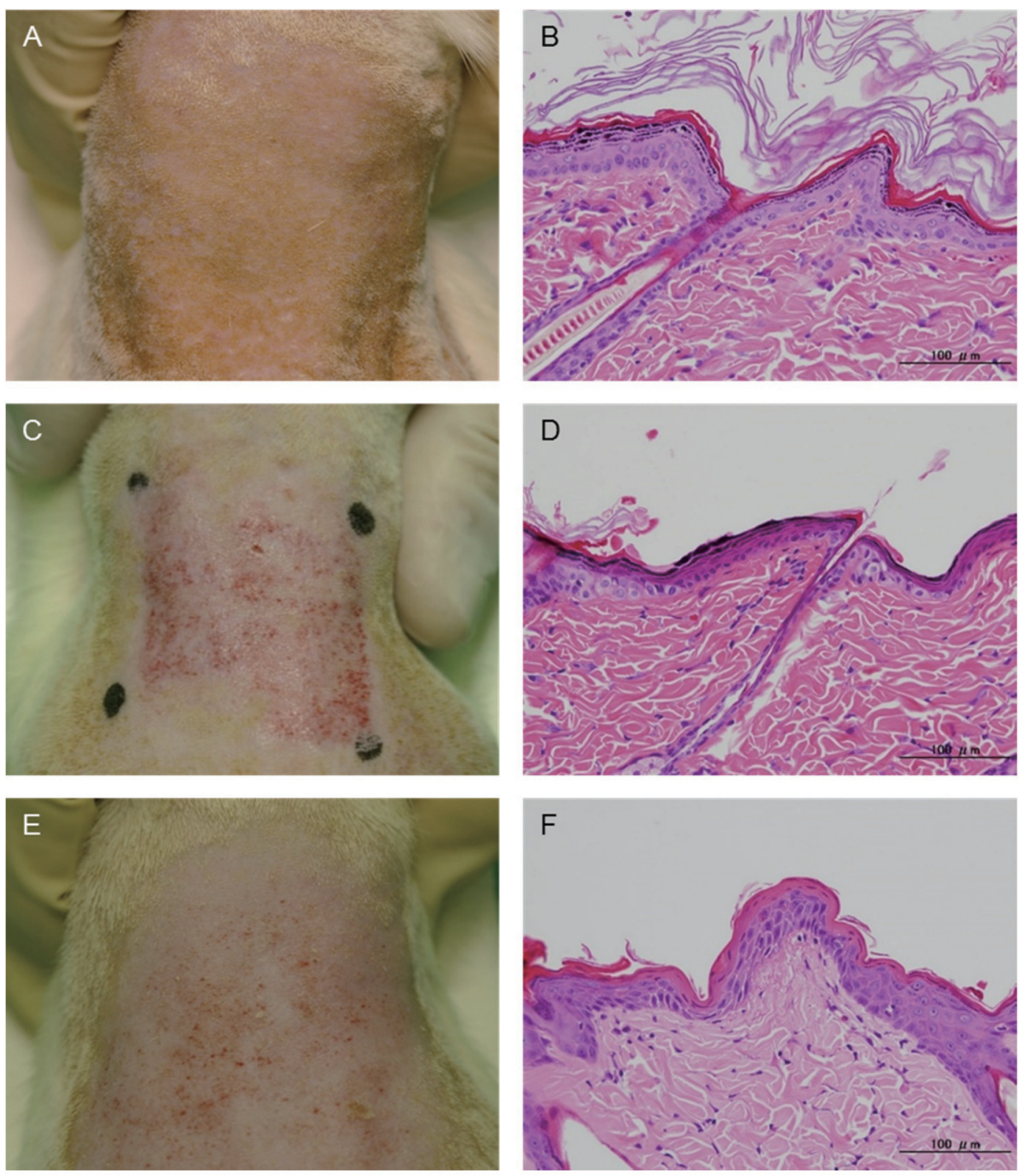

Fig. 3. Dermal changes after skin barrier disruption. Rat skin was shaved and either not pretreated (A, B), pretreated with TS $(\mathrm{C}, \mathrm{D})$ or pretreated with $\mathrm{AC}(\mathrm{E}, \mathrm{F})$. (A, C, E) Macroscopic changes in the skin. (B, D, F) Histopathological changes in the skin.

\section{Morphological changes after skin exposure to silver nanoparticles following TS disruption of skin barrier}

On days 2 and 3, skin to which $5 \mathrm{~nm} \mathrm{Ag} \mathrm{nanoparticles}$ had been applied after TS pretreatment appeared slightly black. This skin discoloration decreased over time, with no macroscopic differences on day 5 among TS-pretreat- ed rats treated percutaneously with $5 \mathrm{~nm}$ silver nanoparticles, $\mathrm{Ag}^{+}$ions and vehicle.

Histopathologic examination on day 2 showed brown pigment, derived from silver, attached to the surface of the epidermis to which $5 \mathrm{~nm} \mathrm{Ag}$ nanoparticles had been applied, with or without TS treatment, and to which $\mathrm{Ag}^{+}$ ions had been applied after TS pretreatment. In the absent 
of TS disruption (5 $\mathrm{nm} \mathrm{Ag} \mathrm{TP-),} \mathrm{there} \mathrm{were} \mathrm{no} \mathrm{other} \mathrm{his-}$ topathological changes of the epidermis or dermis on day 2 . Crust was attached focally to all TS pretreated rats following application of $5 \mathrm{~nm} \mathrm{Ag} \mathrm{nanoparticles,} \mathrm{Ag}^{+}$ions, or vehicle. Rats in these three groups showed thickening of the epidermal layer; parakeratosis, including abnormal cornification and a reduction in keratohyaline granules; and intercellular edema. In addition, the dermis of these three groups showed a very slight infiltration of inflammatory cells, a proliferation of fibroblasts and hemorrhage. All of these changes, however, were comparable in the three groups (Table 1A, Fig. 4A-C). Over time, improvements in histopathology were observed (Table 1A-C, Fig. 4).

\section{Morphological changes after skin exposure to silver nanoparticles following AC disruption of skin barrier}

Rat skin was pretreated for 4 days with AC, following which $5 \mathrm{~nm} \mathrm{Ag} \mathrm{nanoparticles,} \mathrm{Ag}^{+}$ions, or vehicle was applied. Skin to which $\mathrm{Ag}^{+}$ions had been applied appeared slightly black.

Histopathologic examination showed brown pigment, derived from silver, on the surface of the epidermis following treatment with $5 \mathrm{~nm} \mathrm{Ag} \mathrm{nanoparticles} \mathrm{and} \mathrm{Ag}^{+}$ ions. Rats pretreated with $\mathrm{AC}$ showed very slight parakeratosis, with a reduction in keratohyaline granules and thickening of the epidermal layer following application of $5 \mathrm{~nm} \mathrm{Ag}$ nanoparticles, $\mathrm{Ag}^{+}$ions, or vehicle (Table 1D, Fig. 5).
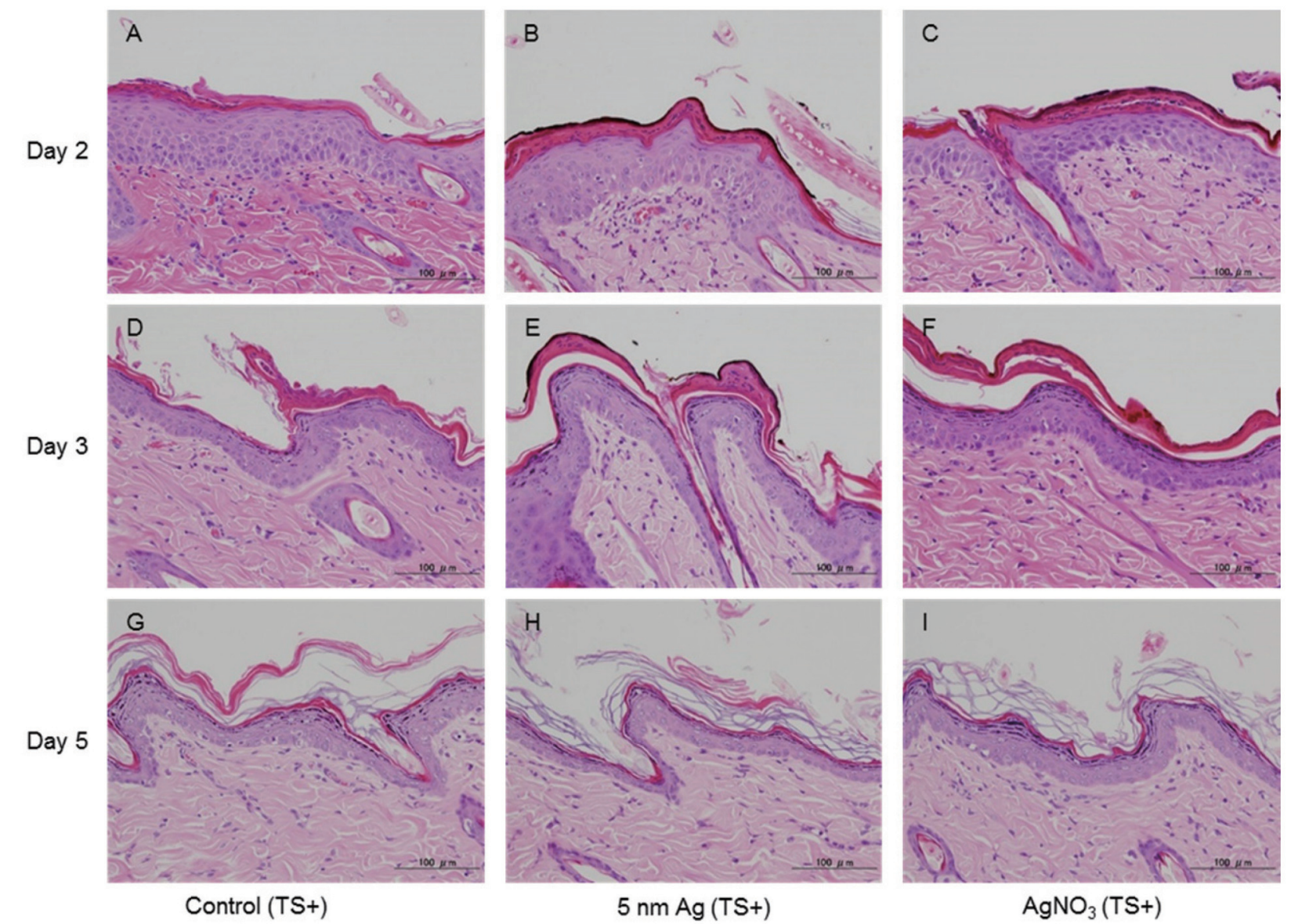

Fig. 4. Dermal histopathological changes after exposure of rat skin to $5 \mathrm{~nm} \mathrm{Ag}$ nanoparticles, $\mathrm{Ag}^{+}$ions, or vehicle following $\mathrm{TS}$ disruption of skin barrier. All rats were pretreated with TS. Skin samples of rats exposed to (A, D, G) vehicle, (B, E, H) $5 \mathrm{~nm}$ Ag nanoparticles, and (C, F, I) Ag $\mathrm{Ag}^{+}$ions were examined after 2 (A-C), 3 (D-F) and 5 (G-I) days. TS+: with tape stripping. 
Table 1. Histopathological findings in male rat skin following exposure to silver nanoparticles for $24 \mathrm{hr}$ after skin barrier disruption by tape stripping (TS) or acetone wiping (AC).

(A) TS group, Day 2

\begin{tabular}{|c|c|c|c|c|c|c|c|c|c|c|c|c|c|c|c|c|c|c|c|c|c|}
\hline \multirow[b]{3}{*}{ Findings } & \multirow{3}{*}{$\begin{array}{l}\text { Group } \\
\text { Pretreatment } \\
\text { Grade }\end{array}$} & \multirow{2}{*}{\multicolumn{5}{|c|}{$\begin{array}{c}\text { Vehicle control } \\
\text { TS }\end{array}$}} & \multicolumn{10}{|c|}{$5 \mathrm{~nm} \mathrm{Ag}(1 \mathrm{mg} / \mathrm{mL})$} & \multirow{2}{*}{\multicolumn{5}{|c|}{$\frac{\mathrm{AgNO}_{3}(1 \mathrm{mg} / \mathrm{mL})}{\mathrm{TS}}$}} \\
\hline & & & & & & & \multicolumn{5}{|c|}{ TS } & \multicolumn{5}{|c|}{ not pretreatment } & & & & & \\
\hline & & - & \pm & + & $2+$ & $3+$ & - & \pm & + & $2+$ & $3+$ & - & \pm & + & $2+$ & $3+$ & - & \pm & + & $2+$ & $3+$ \\
\hline \multicolumn{22}{|l|}{ Epidermis } \\
\hline \multicolumn{2}{|l|}{ Parakeratosis } & 1 & 1 & 2 & 0 & 0 & 0 & 1 & 3 & 0 & 0 & 2 & 0 & 0 & 0 & 0 & 0 & 2 & 2 & 0 & 0 \\
\hline \multicolumn{2}{|c|}{ Decrease, keratohyaline granule } & 1 & 0 & 1 & 2 & 0 & 0 & 1 & 1 & 2 & 0 & 2 & 0 & 0 & 0 & 0 & 0 & 1 & 1 & 2 & 0 \\
\hline \multicolumn{2}{|c|}{ Attachment, brown pigment } & 4 & 0 & 0 & 0 & 0 & 0 & 0 & 4 & 0 & 0 & 1 & 1 & 0 & 0 & 0 & 1 & 3 & 0 & 0 & 0 \\
\hline \multicolumn{2}{|l|}{ Crust, focal } & 1 & 2 & 1 & 0 & 0 & 1 & 2 & 1 & 0 & 0 & 2 & 0 & 0 & 0 & 0 & 0 & 2 & 1 & 1 & 0 \\
\hline \multicolumn{2}{|l|}{ Thickening } & 0 & 4 & 0 & 0 & 0 & 0 & 4 & 0 & 0 & 0 & 2 & 0 & 0 & 0 & 0 & 1 & 3 & 0 & 0 & 0 \\
\hline \multicolumn{2}{|c|}{ Edema, intercellular } & 1 & 3 & 0 & 0 & 0 & 1 & 3 & 0 & 0 & 0 & 2 & 0 & 0 & 0 & 0 & 1 & 3 & 0 & 0 & 0 \\
\hline \multicolumn{22}{|l|}{ Dermis } \\
\hline \multirow{2}{*}{\multicolumn{2}{|c|}{$\begin{array}{l}\text { Cellular infiltration, inflammatory } \\
\text { Proliferation, fibroblast }\end{array}$}} & 1 & 3 & 0 & 0 & 0 & 1 & 2 & 1 & 0 & 0 & 2 & 0 & 0 & 0 & 0 & 1 & 1 & 2 & 0 & 0 \\
\hline & & 0 & 4 & 0 & 0 & 0 & 0 & 4 & 0 & 0 & 0 & 2 & 0 & 0 & 0 & 0 & 0 & 4 & 0 & 0 & 0 \\
\hline \multicolumn{2}{|l|}{ Hemorrhage } & 3 & 1 & 0 & 0 & 0 & 2 & 2 & 0 & 0 & 0 & 2 & 0 & 0 & 0 & 0 & 2 & 2 & 0 & 0 & 0 \\
\hline
\end{tabular}

(B) TS group, Day 3

\begin{tabular}{|c|c|c|c|c|c|c|c|c|c|c|c|c|c|c|c|c|}
\hline \multirow[b]{3}{*}{ Findings } & \multirow{3}{*}{$\begin{array}{l}\text { Group } \\
\text { Pretreatment } \\
\text { Grade }\end{array}$} & \multicolumn{5}{|c|}{ Vehicle control } & \multicolumn{5}{|c|}{$5 \mathrm{~nm} \mathrm{Ag}(1 \mathrm{mg} / \mathrm{mL})$} & \multicolumn{5}{|c|}{$\mathrm{AgNO}_{3}(1 \mathrm{mg} / \mathrm{mL})$} \\
\hline & & \multicolumn{5}{|c|}{ TS } & \multicolumn{5}{|c|}{ TS } & \multicolumn{5}{|c|}{ TS } \\
\hline & & - & \pm & + & $2+$ & $3+$ & - & \pm & + & $2+$ & $3+$ & - & \pm & + & $2+$ & $3+$ \\
\hline \multicolumn{17}{|l|}{ Epidermis } \\
\hline \multicolumn{2}{|l|}{ Parakeratosis } & 0 & 0 & 1 & 1 & 0 & 0 & 1 & 0 & 1 & 0 & 0 & 0 & 2 & 0 & 0 \\
\hline \multicolumn{2}{|c|}{ Decrease, keratohyaline granule } & 1 & 1 & 0 & 0 & 0 & 1 & 1 & 0 & 0 & 0 & 1 & 1 & 0 & 0 & 0 \\
\hline \multicolumn{2}{|c|}{ Attachment, brown pigment } & 2 & 0 & 0 & 0 & 0 & 0 & 1 & 1 & 0 & 0 & 0 & 0 & 2 & 0 & 0 \\
\hline \multicolumn{2}{|l|}{ Crust, focal } & 0 & 0 & 2 & 0 & 0 & 0 & 2 & 0 & 0 & 0 & 0 & 2 & 0 & 0 & 0 \\
\hline \multicolumn{2}{|l|}{ Thickening } & 0 & 2 & 0 & 0 & 0 & 0 & 2 & 0 & 0 & 0 & 0 & 2 & 0 & 0 & 0 \\
\hline \multicolumn{17}{|l|}{ Dermis } \\
\hline \multicolumn{2}{|c|}{ Cellular infiltration, infl } & 1 & 1 & 0 & 0 & 0 & & 0 & 0 & 0 & 0 & 2 & 0 & 0 & 0 & 0 \\
\hline \multicolumn{2}{|c|}{ Proliferation, fibroblast } & 0 & 2 & 0 & 0 & 0 & 1 & 1 & 0 & 0 & 0 & 0 & 2 & 0 & 0 & 0 \\
\hline
\end{tabular}

\section{(C) TS group, Day 5}

\begin{tabular}{|c|c|c|c|c|c|c|c|c|c|c|c|c|c|c|c|c|c|c|c|c|c|}
\hline \multirow[b]{3}{*}{ Findings } & \multirow{3}{*}{$\begin{array}{l}\text { Group } \\
\text { Pretreatment } \\
\text { Grade }\end{array}$} & \multirow{2}{*}{\multicolumn{5}{|c|}{$\begin{array}{c}\text { Vehicle control } \\
\text { TS }\end{array}$}} & \multicolumn{10}{|c|}{$5 \mathrm{~nm} \mathrm{Ag}(1 \mathrm{mg} / \mathrm{mL})$} & \multirow{2}{*}{\multicolumn{5}{|c|}{$\frac{\mathrm{AgNO}_{3}(1 \mathrm{mg} / \mathrm{mL})}{\mathrm{TS}}$}} \\
\hline & & & & & & & \multicolumn{5}{|c|}{$\mathrm{TS}$} & \multicolumn{5}{|c|}{ not pretreatment } & & & & & \\
\hline & & - & \pm & + & $2+$ & $3+$ & - & \pm & + & $2+$ & $3+$ & - & \pm & + & $2+$ & $3+$ & - & \pm & + & $2+$ & $3+$ \\
\hline \multicolumn{22}{|l|}{ Epidermis } \\
\hline \multirow{2}{*}{\multicolumn{2}{|c|}{$\begin{array}{l}\text { Parakeratosis, focal } \\
\text { Decrease, keratohyaline granule, } \\
\text { focal }\end{array}$}} & 1 & 4 & 0 & 0 & 0 & 4 & 1 & 0 & 0 & 0 & 5 & 0 & 0 & 0 & 0 & 2 & 3 & 0 & 0 & 0 \\
\hline & & 5 & 0 & 0 & 0 & 0 & 5 & 0 & 0 & 0 & 0 & 5 & 0 & 0 & 0 & 0 & 4 & 1 & 0 & 0 & 0 \\
\hline \multicolumn{2}{|l|}{ Crust, focal } & 2 & 3 & 0 & 0 & 0 & 4 & 1 & 0 & 0 & 0 & 4 & 1 & 0 & 0 & 0 & 4 & 1 & 0 & 0 & 0 \\
\hline \multicolumn{2}{|l|}{ Thickening, focal } & 3 & 2 & 0 & 0 & 0 & 5 & 0 & 0 & 0 & 0 & 4 & 1 & 0 & 0 & 0 & 4 & 1 & 0 & 0 & 0 \\
\hline \multicolumn{22}{|l|}{ Dermis } \\
\hline \multicolumn{2}{|c|}{ Cellular infiltration, inflammatory, focal } & 4 & 1 & 0 & 0 & 0 & 5 & 0 & 0 & 0 & 0 & 5 & 0 & 0 & 0 & 0 & 5 & 0 & 0 & 0 & 0 \\
\hline \multicolumn{2}{|c|}{ Proliferation, fibroblast, focal } & 4 & 1 & 0 & 0 & 0 & 5 & 0 & 0 & 0 & 0 & 5 & 0 & 0 & 0 & 0 & 3 & 2 & 0 & 0 & 0 \\
\hline
\end{tabular}

(D) AC group, Day 2

\begin{tabular}{|c|c|c|c|c|c|c|c|c|c|c|c|c|c|c|c|c|}
\hline \multirow[b]{3}{*}{ Findings } & \multirow{3}{*}{$\begin{array}{l}\text { Group } \\
\text { Pretreatment } \\
\text { Grade }\end{array}$} & \multicolumn{5}{|c|}{ Vehicle control } & \multicolumn{5}{|c|}{$5 \mathrm{~nm} \mathrm{Ag}(1 \mathrm{mg} / \mathrm{mL})$} & \multicolumn{5}{|c|}{$\mathrm{AgNO}_{3}(1 \mathrm{mg} / \mathrm{mL})$} \\
\hline & & \multicolumn{5}{|c|}{$\mathrm{AC}$} & \multicolumn{5}{|c|}{$\mathrm{AC}$} & \multicolumn{5}{|c|}{$\mathrm{AC}$} \\
\hline & & - & \pm & + & $2+$ & $3+$ & - & \pm & + & $2+$ & $3+$ & - & \pm & + & $2+$ & $3+$ \\
\hline \multicolumn{17}{|l|}{ Epidermis } \\
\hline \multicolumn{2}{|l|}{ Parakeratosis, focal } & 2 & 2 & 0 & 0 & 0 & 1 & 3 & 0 & 0 & 0 & 1 & 3 & 0 & 0 & 0 \\
\hline \multicolumn{2}{|c|}{ Decrease, keratohyaline granule } & 3 & 1 & 0 & 0 & 0 & 2 & 2 & 0 & 0 & 0 & 3 & 1 & 0 & 0 & 0 \\
\hline \multicolumn{2}{|c|}{ Attachment, brown pigment } & 4 & 0 & 0 & 0 & 0 & 1 & 2 & 1 & 0 & 0 & 0 & 4 & 0 & 0 & 0 \\
\hline \multicolumn{2}{|l|}{ Crust, focal } & 3 & 1 & 0 & 0 & 0 & 4 & 0 & 0 & 0 & 0 & 4 & 0 & 0 & 0 & 0 \\
\hline \multicolumn{2}{|l|}{ Thickening } & 0 & 4 & 0 & 0 & 0 & 0 & 4 & 0 & 0 & 0 & 0 & 4 & 0 & 0 & 0 \\
\hline \multicolumn{17}{|l|}{ Dermis } \\
\hline \multicolumn{2}{|c|}{ Proliferation, fibroblast, focal } & 3 & 1 & 0 & 0 & 0 & 4 & 0 & 0 & 0 & 0 & 4 & 0 & 0 & 0 & 0 \\
\hline
\end{tabular}

Notes) - : No abnormal changes \pm : Very slight + : Slight $2+$ : Moderate $3+$ : Marked

Numerals represent the number of animals.

Vehicle control: $2 \mathrm{mM}$ citric acid solution

TS: Tape stripping

AC: Acetone wiping

Vol. 4 No. 3 

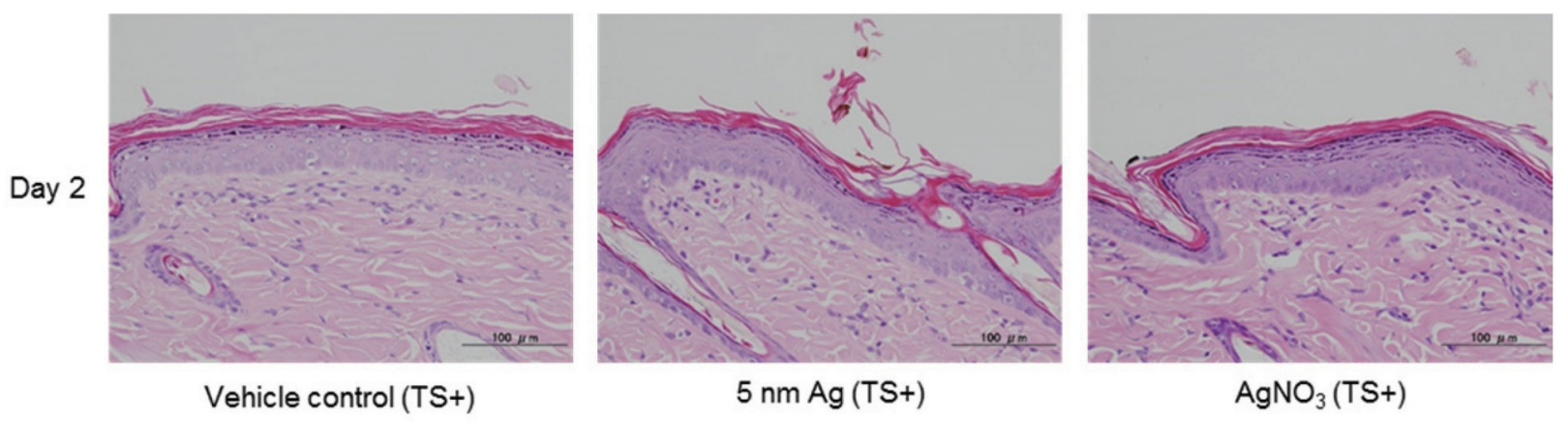

Fig. 5. Dermal histopathological changes after exposure of rat skin to $5 \mathrm{~nm} \mathrm{Ag}$ nanoparticles, $\mathrm{Ag}^{+}$ions, or vehicle following $\mathrm{AC}$ disruption of skin barrier for 4 days. Skin samples were obtained the day after exposure of rats to (A) vehicle, (B) $5 \mathrm{~nm} \mathrm{Ag}$ nanoparticles, and $(\mathrm{C}) \mathrm{Ag}^{+}$ions. TS+: with tape stripping.

Table 2. ICP-MS analysis of male rats exposed to silver nanoparticles for $24 \mathrm{hr}$ after skin barrier disruptions by tape stripping (TS) or acetone wiping (AC).

\begin{tabular}{|c|c|c|c|c|c|c|c|}
\hline \multirow[b]{2}{*}{ Pretreatment } & \multirow[b]{2}{*}{ Group } & \multicolumn{2}{|c|}{ Skin } & \multicolumn{2}{|c|}{ Liver } & \multicolumn{2}{|c|}{ Blood } \\
\hline & & Day 2 & Day 5 & Day 2 & Day 5 & Day 2 & Day 5 \\
\hline \multirow[t]{3}{*}{ Not pretreatment } & Vehicle control & $<0.05$ & - & $<0.05$ & - & $<0.05$ & - \\
\hline & $5 \mathrm{~nm} \mathrm{Ag}(1 \mathrm{mg} / \mathrm{mL})$ & 9.7 & - & $<0.05$ & - & $<0.05$ & - \\
\hline & $\mathrm{AgNO}_{3}(1 \mathrm{mg} / \mathrm{mL})$ & 2.5 & - & $<0.05$ & - & $<0.05$ & - \\
\hline \multirow[t]{3}{*}{ TS } & Vehicle control & $<0.05$ & $<0.05$ & $<0.05$ & $<0.05$ & $<0.05$ & $<0.05$ \\
\hline & $5 \mathrm{~nm} \mathrm{Ag}(1 \mathrm{mg} / \mathrm{mL})$ & 63.0 & 26.5 & $<0.05$ & $<0.05$ & $<0.05$ & $<0.05$ \\
\hline & $\mathrm{AgNO}_{3}(1 \mathrm{mg} / \mathrm{mL})$ & 88.0 & 13.4 & 0.12 & 0.07 & $<0.05$ & $<0.05$ \\
\hline \multirow[t]{3}{*}{$\mathrm{AC}$} & Vehicle control & $<0.05$ & - & $<0.05$ & - & $<0.05$ & - \\
\hline & $5 \mathrm{~nm} \mathrm{Ag}(1 \mathrm{mg} / \mathrm{mL})$ & 97.5 & - & $<0.05$ & - & $<0.05$ & - \\
\hline & $\mathrm{AgNO}_{3}(1 \mathrm{mg} / \mathrm{mL})$ & 25.0 & - & $<0.05$ & - & $<0.05$ & - \\
\hline
\end{tabular}

Each value shows mean (ppm).

The lower limits of Ag detection : $0.05 \mathrm{ppm}$.

- : Not examined.

Vehicle control: $2 \mathrm{mM}$ citric acid solution

TS: Tape stripping

$\mathrm{AC}$ : Acetone wiping

\section{Ag distribution in tissues}

Ag was detected in skin of all rats, except the control group, regardless of whether they had been pretreated with TS or AC or had not been pretreated. The amount of Ag was higher in rats pretreated with TS or AC than in those without pretreatment. In the blood, Ag was under the limit of detection $(<0.05 \mathrm{ppm})$ in all groups. In the liver, Ag was detected only in rats treated with both TS and $\mathrm{Ag}^{+}$ions. $\mathrm{Ag}$ contents in the skin and liver decreased over time, but were still present on day 5 (Table 2).

\section{Systemic toxicities of silver nanoparticles following skin barrier disruption with TS}

Macroscopic examination showed that neither $5 \mathrm{~nm} \mathrm{Ag}$ nanoparticles nor $\mathrm{Ag}^{+}$ions had any adverse effects over time on rats that were and were not pretreated with TS. Body weighs and weights of the liver, spleen and adrenal glands on day 5 were similar among rats treated with
$5 \mathrm{~nm} \mathrm{Ag}$ nanoparticles, $\mathrm{Ag}^{+}$ions, and vehicle with or without TS pretreatment. In contrast, relative kidney weight was lower in rats treated with $5 \mathrm{~nm} \mathrm{Ag}$ nanoparticles and $\mathrm{Ag}^{+}$ions following pretreatment with TS (Table 3).

Histopathological examinations of the liver, kidneys, spleen and adrenal glands showed that neither $5 \mathrm{~nm} \mathrm{Ag}$ nanoparticles nor $\mathrm{Ag}^{+}$ions had any adverse effects, with or without TS (Table 4).

Hematological analyses showed minor alterations in rats treated with $5 \mathrm{~nm} \mathrm{Ag}$ nanoparticles and $\mathrm{Ag}^{+}$ions following TS. Mean corpuscular cell volume (MCV) and corpuscular cell hemoglobin (MCH) were increased, without any changes in red blood cell count, hemoglobin concentration, and hematocrit (Table 5). Glucose concentration was lower in rats treated with $\mathrm{Ag}^{+}$ions after TS disruption, but there were no other changes in clinical chemistry following treated with $5 \mathrm{~nm} \mathrm{Ag}$ nanoparticles or $\mathrm{Ag}^{+}$ions with or without TS disruption (Table 6). 
Table 3. Organ weights of male rats exposed to silver nanoparticles for $24 \mathrm{hr}$ after skin barrier disruption by tape stripping (TS).

\begin{tabular}{|c|c|c|c|c|c|}
\hline Group & & Vehicle control & $5 \mathrm{~nm} \mathrm{~A}$ & $\mathrm{~g} / \mathrm{mL})$ & $\mathrm{AgNO}_{3}(1 \mathrm{mg} / \mathrm{mL})$ \\
\hline Pretreatment & & TS & TS & not pretreatment & $\mathrm{TS}$ \\
\hline Number of animals & & 5 & 5 & 5 & 5 \\
\hline Body weight & (g) & $242.2 \pm 9.6$ & $247.9 \pm 11.1$ & $247.6 \pm 4.4$ & $250.8 \pm 9.3$ \\
\hline Liver & $\begin{array}{c}(\mathrm{mg}) \\
(\mathrm{mg} / \mathrm{g})\end{array}$ & $\begin{array}{l}8729.8 \pm 1054.2 \\
36.015 \pm 3.646\end{array}$ & $\begin{array}{l}8653.1 \pm 602.0 \\
34.889 \pm 1.150\end{array}$ & $\begin{array}{l}8202.4 \pm 629.2 \\
33.102 \pm 2.060\end{array}$ & $\begin{array}{l}8292.3 \pm 499.8 \\
33.111 \pm 2.509\end{array}$ \\
\hline Kidneys & $\begin{array}{c}(\mathrm{mg}) \\
(\mathrm{mg} / \mathrm{g})\end{array}$ & $\begin{array}{l}2472.0 \pm 178.1 \\
10.202 \pm 0.518\end{array}$ & $\begin{aligned} 2208.7 & \pm 196.0 \\
8.903 & \pm 0.527^{* *}\end{aligned}$ & $\begin{aligned} 2339.9 & \pm 88.1 \\
9.448 & \pm 0.280 *\end{aligned}$ & $\begin{aligned} 2342.9 & \pm 163.9 \\
9.340 & \pm 0.525 *\end{aligned}$ \\
\hline Spleen & $\begin{array}{c}(\mathrm{mg}) \\
(\mathrm{mg} / \mathrm{g})\end{array}$ & $\begin{array}{l}703.2 \pm 175.5 \\
2.890 \pm 0.633\end{array}$ & $\begin{array}{l}716.0 \pm 121.5 \\
2.889 \pm 0.464\end{array}$ & $\begin{array}{l}657.5 \pm 79.9 \\
2.657 \pm 0.336\end{array}$ & $\begin{array}{l}669.9 \pm 110.8 \\
2.662 \pm 0.359\end{array}$ \\
\hline Adrenal glands & $\begin{array}{c}(\mathrm{mg}) \\
(\mathrm{mg} / \mathrm{g})\end{array}$ & $\begin{aligned} 53.8 & \pm 7.6 \\
0.222 & \pm 0.027\end{aligned}$ & $\begin{aligned} 48.2 & \pm 6.6 \\
0.194 & \pm 0.024\end{aligned}$ & $\begin{aligned} 51.2 & \pm 3.4 \\
0.207 & \pm 0.014\end{aligned}$ & $\begin{aligned} 52.8 & \pm 3.6 \\
0.210 & \pm 0.015\end{aligned}$ \\
\hline
\end{tabular}

Each value shows mean \pm S.D.

Significantly different from the control group $(*: \mathrm{P}<0.05, * *: \mathrm{P}<0.01)$.

Vehicle control: $2 \mathrm{mM}$ citric acid solution

TS: Tape stripping

Table 4. Histopathological findings of male rats exposed to silver nanoparticles for $24 \mathrm{hr}$ after skin barrier disruption by tape stripping (TS).

\begin{tabular}{|c|c|c|c|c|c|c|c|c|c|c|c|c|c|c|c|c|c|c|c|c|c|}
\hline \multirow[b]{3}{*}{ Findings } & \multirow{3}{*}{$\begin{array}{l}\text { Group } \\
\text { Pretreatment } \\
\text { Grade }\end{array}$} & \multirow{2}{*}{\multicolumn{5}{|c|}{$\begin{array}{c}\text { Vehicle control } \\
\text { TS }\end{array}$}} & \multicolumn{10}{|c|}{$5 \mathrm{~nm} \mathrm{Ag}(1 \mathrm{mg} / \mathrm{mL})$} & \multirow{2}{*}{\multicolumn{5}{|c|}{$\begin{array}{c}\mathrm{AgNO}_{3}(1 \mathrm{mg} / \mathrm{mL}) \\
\mathrm{TS}\end{array}$}} \\
\hline & & & & & & & \multicolumn{5}{|c|}{ TS } & \multicolumn{5}{|c|}{ not preteatmnet } & & & & & \\
\hline & & - & \pm & + & $2+$ & $3+$ & - & \pm & + & $2+$ & $3+$ & - & \pm & + & $2+$ & $3+$ & - & \pm & + & $2+$ & $3+$ \\
\hline \multicolumn{22}{|l|}{ Liver } \\
\hline \multicolumn{2}{|c|}{ Cellular infiltration, mixed, focal } & 5 & 0 & 0 & 0 & 0 & 4 & 1 & 0 & 0 & 0 & 5 & 0 & 0 & 0 & 0 & 5 & 0 & 0 & 0 & 0 \\
\hline \multicolumn{2}{|c|}{ Fatty change, hepatocyte, periportal } & 4 & 1 & 0 & 0 & 0 & 4 & 1 & 0 & 0 & 0 & 4 & 1 & 0 & 0 & 0 & 4 & 1 & 0 & 0 & 0 \\
\hline \multicolumn{2}{|c|}{ Hypertrophy/hyperplasia, Kupffer cell } & 4 & 1 & 0 & 0 & 0 & 5 & 0 & 0 & 0 & 0 & 5 & 0 & 0 & 0 & 0 & 5 & 0 & 0 & 0 & 0 \\
\hline \multicolumn{22}{|l|}{ Kidney } \\
\hline \multicolumn{2}{|c|}{ Basophilic tubule, cortex } & 2 & 3 & 0 & 0 & 0 & 2 & 3 & 0 & 0 & 0 & 2 & 3 & 0 & 0 & 0 & 3 & 1 & 1 & 0 & 0 \\
\hline \multicolumn{2}{|c|}{ Cast, hyalin, medulla, unilateral } & 5 & 0 & 0 & 0 & 0 & 4 & 1 & 0 & 0 & 0 & 5 & 0 & 0 & 0 & 0 & 5 & 0 & 0 & 0 & 0 \\
\hline \multicolumn{2}{|c|}{ Dilatation, pelvis, unilateral } & 5 & 0 & 0 & 0 & 0 & 3 & 0 & 1 & 1 & 0 & 5 & 0 & 0 & 0 & 0 & 5 & 0 & 0 & 0 & 0 \\
\hline \multicolumn{22}{|l|}{ Spleen } \\
\hline \multicolumn{2}{|c|}{ Hematopoiesis, extramedullary } & 0 & 0 & 4 & 1 & 0 & 0 & 0 & 3 & 2 & 0 & 0 & 0 & 3 & 2 & 0 & 0 & 0 & 5 & 0 & 0 \\
\hline \multicolumn{2}{|c|}{ Adrenal gland } & 5 & & & & & 5 & & & & & 5 & & & & & 5 & & & & \\
\hline
\end{tabular}

Notes) - : No abnormal changes \pm : Very slight + : Slight 2+: Moderate 3+: Marked

Numerals represent the number of animals.

Not significantly different from control.

Vehicle control: $2 \mathrm{mM}$ citric acid solution

TS: Tape stripping

\section{DISCUSSION}

This study, which investigated whether skin was permeable to $5 \mathrm{~nm} \mathrm{Ag}$ nanoparticles following skin barrier disruption with $\mathrm{TS}$ or $\mathrm{AC}$, found that these nanoparticles were unable to penetrate the skin even after pretreatment with TS or AC. Although skin exposed to $5 \mathrm{~nm} \mathrm{Ag}$ nanoparticles after epidermal barrier disruption showed thickening of the epidermal layers and parakeratosis, as well as, a very slight inflammatory response, these histopathological changes were comparable in skin exposed to nanoparticles and vehicle, indicating that the $5 \mathrm{~nm} \mathrm{Ag}$ nanoparticles did not exacerbate epidermal damage morphologically. After exposure Ag nanoparticles to rat skin following TS or AC pretreatment, silver was not detected in liver or blood, indicating that these nanoparticles did not penetrate through the skin even after impairment of the skin barrier. 
Nano-silver permeability of rat skin

Table 5. Hematological findings of male rats exposed to silver nanoparticles after $24 \mathrm{hr}$ after skin barrier disruption by tape stripping (TS).

\begin{tabular}{|c|c|c|c|c|c|}
\hline \multirow{2}{*}{$\begin{array}{l}\text { Group } \\
\text { Pretreatment }\end{array}$} & & \multirow{2}{*}{$\begin{array}{c}\text { Vehicle control } \\
\text { TS }\end{array}$} & \multicolumn{2}{|c|}{$5 \mathrm{~nm} \mathrm{Ag}(1 \mathrm{mg} / \mathrm{mL})$} & \multirow{2}{*}{$\begin{array}{c}\mathrm{AgNO}_{3}(1 \mathrm{mg} / \mathrm{mL}) \\
\mathrm{TS}\end{array}$} \\
\hline & & & TS & not pretreatment & \\
\hline \multicolumn{2}{|c|}{ Number of animals } & 5 & 5 & 5 & 5 \\
\hline $\mathrm{RBC}$ & $10000 / \mu \mathrm{L}$ & $697 \pm 33$ & $678 \pm 23$ & $714 \pm 32$ & $665 \pm 28$ \\
\hline Hemoglobin & $\mathrm{g} / \mathrm{dL}$ & $14.3 \pm 0.7$ & $14.8 \pm 0.3$ & $15.2 \pm 0.6$ & $14.4 \pm 0.4$ \\
\hline Hematocrit & $\%$ & $41.4 \pm 1.5$ & $42.9 \pm 1.0$ & $43.7 \pm 1.9$ & $42.1 \pm 1.1$ \\
\hline $\mathrm{MCV}$ & $\mathrm{fL}$ & $59.5 \pm 1.8$ & $63.2 \pm 1.6 * *$ & $61.3 \pm 1.2$ & $63.4 \pm 1.5 * *$ \\
\hline $\mathrm{MCH}$ & pg & $20.5 \pm 0.7$ & $21.8 \pm 0.6 * *$ & $21.3 \pm 0.4$ & $21.7 \pm 0.4 * *$ \\
\hline $\mathrm{MCHC}$ & $\mathrm{g} / \mathrm{dL}$ & $34.4 \pm 0.4$ & $34.5 \pm 0.4$ & $34.7 \pm 0.2$ & $34.2 \pm 0.3$ \\
\hline Platelet & $10000 / \mu \mathrm{L}$ & $134.1 \pm 14.0$ & $126.8 \pm 16.4$ & $105.6 \pm 42.9$ & $116.3 \pm 9.4$ \\
\hline WBC & $100 / \mu \mathrm{L}$ & $116.3 \pm 32.3$ & $111.5 \pm 24.3$ & $89.8 \pm 10.4$ & $88.6 \pm 40.0$ \\
\hline Neutrophil & $\%$ & $10.2 \pm 1.5$ & $13.9 \pm 8.7$ & $13.9 \pm 4.2$ & $10.4 \pm 1.5$ \\
\hline Eosinophil & $\%$ & $0.8 \pm 0.3$ & $0.9 \pm 0.3$ & $0.8 \pm 0.1$ & $0.9 \pm 0.4$ \\
\hline Basophil & $\%$ & $0.1 \pm 0.0$ & $0.0 \pm 0.0$ & $0.0 \pm 0.0$ & $0.1 \pm 0.1$ \\
\hline Monocyte & $\%$ & $2.7 \pm 0.4$ & $2.6 \pm 0.9$ & $2.6 \pm 1.1$ & $2.4 \pm 0.6$ \\
\hline Lymphocyte & $\%$ & $86.2 \pm 1.8$ & $82.6 \pm 9.4$ & $82.7 \pm 5.2$ & $86.2 \pm 1.9$ \\
\hline Reticulocyte & $\%$ & $5.42 \pm 0.52$ & $5.16 \pm 1.22$ & $5.66 \pm 0.88$ & $5.39 \pm 0.77$ \\
\hline
\end{tabular}

Each value shows mean \pm S.D.

Significantly different from the control group (**: $\mathrm{P}<0.01)$.

Vehicle control: $2 \mathrm{mM}$ citric acid solution

TS: Tape stripping

Table 6. Blood chemistry of male rats exposed to silver nanoparticles for $24 \mathrm{hr}$ after skin barrier disruption by tape stripping (TS).

\begin{tabular}{|c|c|c|c|c|c|}
\hline \multirow{2}{*}{$\begin{array}{l}\text { Group } \\
\text { Pretreatment }\end{array}$} & & \multicolumn{2}{|c|}{ Vehicle control } & \multirow{2}{*}{$\begin{array}{c}5 \mathrm{~nm} \mathrm{Ag}(1 \mathrm{mg} / \mathrm{mL}) \\
\text { not pretreatment }\end{array}$} & \multirow{2}{*}{$\frac{\mathrm{AgNO}_{3}(1 \mathrm{mg} / \mathrm{mL})}{\mathrm{TS}}$} \\
\hline & & TS & TS & & \\
\hline Number of animals & & 5 & 5 & 5 & 5 \\
\hline Total protein & $\mathrm{g} / \mathrm{dL}$ & $4.9 \pm 0.1$ & $4.9 \pm 0.2$ & $4.9 \pm 0.2$ & $4.8 \pm 0.2$ \\
\hline Albumin & $\mathrm{g} / \mathrm{dL}$ & $3.2 \pm 0.1$ & $3.2 \pm 0.1$ & $3.2 \pm 0.2$ & $3.1 \pm 0.2$ \\
\hline $\mathrm{A} / \mathrm{G}$ & & $1.96 \pm 0.26$ & $1.84 \pm 0.10$ & $1.86 \pm 0.10$ & $1.86 \pm 0.16$ \\
\hline Glucose & $\mathrm{mg} / \mathrm{dL}$ & $144 \pm 5$ & $137 \pm 13$ & $133 \pm 12$ & $114 \pm 15 * *$ \\
\hline Total cholesterol & $\mathrm{mg} / \mathrm{dL}$ & $44 \pm 7$ & $49 \pm 8$ & $49 \pm 7$ & $46 \pm 10$ \\
\hline Triglyceride & $\mathrm{mg} / \mathrm{dL}$ & $22 \pm 6$ & $35 \pm 16$ & $22 \pm 18$ & $17 \pm 5$ \\
\hline AST & $\mathrm{U} / \mathrm{L}$ & $64 \pm 6$ & $67 \pm 8$ & $66 \pm 7$ & $63 \pm 9$ \\
\hline ALT & $\mathrm{U} / \mathrm{L}$ & $23 \pm 5$ & $27 \pm 3$ & $24 \pm 6$ & $23 \pm 2$ \\
\hline BUN & $\mathrm{mg} / \mathrm{dL}$ & $12 \pm 2$ & $12 \pm 3$ & $11 \pm 1$ & $11 \pm 1$ \\
\hline Creatinine & $\mathrm{mg} / \mathrm{dL}$ & $0.21 \pm 0.01$ & $0.21 \pm 0.02$ & $0.20 \pm 0.02$ & $0.20 \pm 0.02$ \\
\hline ALP & $\mathrm{U} / \mathrm{L}$ & $1030 \pm 191$ & $1160 \pm 148$ & $1265 \pm 173$ & $1064 \pm 113$ \\
\hline Inorganic phosphorus & $\mathrm{mg} / \mathrm{dL}$ & $8.1 \pm 0.3$ & $8.3 \pm 0.5$ & $8.2 \pm 0.5$ & $8.4 \pm 0.3$ \\
\hline $\mathrm{Ca}$ & $\mathrm{mg} / \mathrm{dL}$ & $9.1 \pm 0.3$ & $9.3 \pm 0.2$ & $9.2 \pm 0.2$ & $9.5 \pm 0.3$ \\
\hline $\mathrm{Na}$ & $\mathrm{mEq} / \mathrm{L}$ & $145.2 \pm 1.9$ & $146.8 \pm 1.2$ & $146.7 \pm 0.9$ & $147.7 \pm 1.3$ \\
\hline $\mathrm{K}$ & $\mathrm{mEq} / \mathrm{L}$ & $4.09 \pm 0.22$ & $4.04 \pm 0.10$ & $4.20 \pm 0.20$ & $4.06 \pm 0.24$ \\
\hline $\mathrm{Cl}$ & $\mathrm{mEq} / \mathrm{L}$ & $102.2 \pm 0.3$ & $102.4 \pm 0.8$ & $102.6 \pm 2.0$ & $103.6 \pm 1.4$ \\
\hline
\end{tabular}

Each value shows mean \pm S.D.

Significantly different from the control group $(* *: \mathrm{P}<0.01)$.

Vehicle control: $2 \mathrm{mM}$ citric acid solution

TS: Tape stripping

Previous studies demonstrated that following intravenious injections into rats of 20-110 nm silver nanoparticles, whether once or five consecutive days, the nanoparticles disappeared rapidly from the blood circulation, and were distributed mainly to the liver, spleen and kid- neys (Lankveld et al., 2010). Twenty-four weeks after subcutaneous injection of 50-100 nm silver nanoparticles $(62.8 \mathrm{mg} / \mathrm{kg})$ into rats, silver was observed in the blood, kidneys, liver and spleen at the end of study (Tang et al., 2009). These two studies showed that silver nanoparti- 
cles mainly accumulated in the liver and kidneys, and that silver nanoparticles were excreted primarily through the feces.

In this study, only $\mathrm{Ag}^{+}$ions, not $5 \mathrm{~nm} \mathrm{Ag}$ nanoparticles, reached the liver through skin damaged by TS. However, the mount of $\mathrm{Ag}$ in the liver was very small, and tended to decrease over time, providing further evidence that $\mathrm{Ag}$ does not accumulate in the liver. Blood glucose concentrations were reduced following exposure to $\mathrm{Ag}^{+}$ions. The liver produces, stores, and releases glucose depending on bodily need (Treinen-Moslen, 2001). We found that liver weight and hepatic histopathology were not affected by exposure to $5 \mathrm{~nm} \mathrm{Ag}$ nanoparticles or $\mathrm{Ag}^{+}$ions. To prevent cross contamination of $5 \mathrm{~nm} \mathrm{Ag}$ nanoparticles and $\mathrm{Ag}^{+}$ions, animals exposed to $\mathrm{Ag}^{+}$ions were autopsied after those exposed to vehicle and Ag nanoparticles. Thus, the fasting time in rats exposed to $\mathrm{Ag}^{+}$ions was relatively longer than in control rats, indicating that the reduction in glucose concentrations was not caused by $\mathrm{Ag}^{+}$ ions. Although relative kidney weights were significantly lower in rats exposed to $5 \mathrm{~nm} \mathrm{Ag} \mathrm{nanoparticles,} \mathrm{with} \mathrm{and}$ without skin disruption by TS, and in rats exposed to $\mathrm{Ag}^{+}$ ions following pretreatment with TS, biological analysis and renal histopathological examination showed no evidence for kidney toxicity of $5 \mathrm{~nm} \mathrm{Ag}$ nanoparticles or $\mathrm{Ag}^{+}$ ions. Taken together, these results indicate that neither $5 \mathrm{~nm} \mathrm{Ag} \mathrm{nanoparticles} \mathrm{nor} \mathrm{Ag}^{+}$ions had any adverse effects on clinical signs, body weight, or hematological or biochemical parameters.

Silver nanoparticles have been reported to induce oxidase stress, DNA damage and apoptosis. Studies in human cell lines, such as dermal fibroblasts (Avalos et al., 2014) and skin carcinoma cells (Kim and Ryu, 2013), and in vivo studies in non-mammalian species, such as zebrafishes and flies (Ahamed et al., 2010), found that silver nanoparticles stimulated oxidative stress, with smaller nanoparticles being much more cytotoxic than larger nanoparticles. In addition, dose-dependent histopathological changes were seen in the skin, liver, and spleen of guinea pigs after dermal exposure to silver nanoparticles $<100$ $\mathrm{nm}$ in diameter (Korani et al., 2011). Our results indicated that $5 \mathrm{~nm} \mathrm{Ag}$ nanoparticles did not induce skin injury including cell death, or any adverse histopathological changes in the liver and spleen, even following disruption of the epidermal barrier. Discrepancies between our study and previous observations may have been due to differences in nanoparticle size, skin structure, and/or species. We also found that, unlike $\mathrm{Ag}^{+}$ions, $5 \mathrm{~nm} \mathrm{Ag} \mathrm{nanoparti-}$ cles did not pass through damaged skin.

Silver ions released from silver formulations are potent antimicrobial agents and, even at very low concentra- tions, can bind to damage bacterial cells at multiple sites (Ahamed et al., 2010; Brandt et al., 2012; Loeschner et al., 2011). The antibacterial activity of silver nanoparticles is also due to $\mathrm{Ag}^{+}$ions (Lok et al., 2007), which readily form on the nanoparticle surface due to reaction with oxygen. Thus, ionization of silver nanoparticles and ability of $\mathrm{Ag}^{+}$ions to penetrate into or across the skin may result in nanotoxicity. Clinical trials have started, but their scope in limited (Bianco et al., 2015b; Munger et al., 2014; Rigo et al., 2013)

In conclusion, this study found that $5 \mathrm{~nm} \mathrm{Ag} \mathrm{nanopar-}$ ticles cannot pass through rat skin, even after disruption of the epidermal barrier. In contrast, $\mathrm{Ag}^{+}$ions were able to penetrate rat skin after epidermal barrier disruption. These findings indicate that, despite the ability of ionized silver nanoparticles to enter tissue, these nanoparticles have no acute toxicity.

\section{ACKNOWLEDGMENTS}

This study was supported by Health Labour Sciences Research Grants from the Ministry of Health, Labour, and Welfare of Japan. The authors thank the staff of the Laboratories of Toxicology and Pathology of Hatano Research Institute, Food and Drug Safety Center, for their technical support.

Conflict of interest---- The authors declare that there is no conflict of interest.

\section{REFERENCES}

Ahamed, M., Alsalhi, M.S. and Siddiqui, M.K. (2010): Silver nanoparticle applications and human health. Clinica Chimica Acta, 411, 1841-1848.

Avalos, A., Haza, A.I., Mateo, D. and Morales, P. (2014): Interactions of manufactured silver nanoparticles of different sizes with normal human dermal fibroblasts. Int. Wound J., 13, 101-109.

Bianco, C., Kezic, S., Crosera, M., Svetlicic, V., Segota, S., Maina, G., Romano, C., Larese, F. and Adami, G. (2015a): In vitro percutaneous penetration and characterization of silver from silvercontaining textiles. Int. J. Nanomedicine, 10, 1899-1908.

Bianco, C., Kezic, S., Visser, M.J., Pluut, O., Adami, G. and Krystek, P. (2015b): Pilot study on the identification of silver in skin layers and urine after dermal exposure to a functionalized textile. Talanta, 136, 23-28.

Brandt, O., Mildner, M., Egger, A.E., Groessl, M., Rix, U., Posch, M., Keppler, B.K., Strupp, C., Mueller, B. and Stingl, G. (2012): Nanoscalic silver possesses broad-spectrum antimicrobial activities and exhibits fewer toxicological side effects than silver sulfadiazine. Nanomedicine, 8, 478-488.

Brouwer, D.H., Spaan, S., Roff, M., Sleeuwenhoek, A., Tuinman, I., Goede, H., van Duuren-Stuurman, B., Filon, F.L., Bello, D. and Cherrie, J.W. (2016): Occupational dermal exposure to nanoparticles and nano-enabled products: Part 2, exploration of expo- 
Nano-silver permeability of rat skin

sure processes and methods of assessment. Int. J. Hyg. Environ. Health, 219, 503-512.

Chen, X. and Schluesener, H.J. (2008): Nanosilver: a nanoproduct in medical application. Toxicol. Lett., 176, 1-12.

Crosera, M., Bovenzi, M., Maina, G., Adami, G., Zanette, C., Florio, C. and Filon Larese, F. (2009): Nanoparticle dermal absorption and toxicity: a review of the literature. Int. Arch. Occup. Environ. Health, 82, 1043-1055.

De Jong, W.H., Van Der Ven, L.T.M., Sleijffers, A., Park, M.V.D.Z., Jansen, E.H.J.M., Van Loveren, H. and Vandebriel, R.J. (2013): Systemic and immunotoxicity of silver nanoparticles in an intravenous 28 days repeated dose toxicity study in rats. Biomaterials, 34, 8333-8343.

Gujjar, M. and Banga, A.K. (2014): Vehicle influence on permeation through intact and compromised skin. Int. J. Pharm., 472, 362-368.

Juling, S., Bachler, G., von Gotz, N., Lichtenstein, D., Bohmert, L., Niedzwiecka, A., Selve, S., Braeuning, A. and Lampen, A. (2016): In vivo distribution of nanosilver in the rat: The role of ions and de novo-formed secondary particles. Food Chem. Toxicol., 97, 327-335.

Kim, S. and Ryu, D.Y. (2013): Silver nanoparticle-induced oxidative stress, genotoxicity and apoptosis in cultured cells and animal tissues. J. Appl. Toxicol., 33, 78-89.

Kim, Y.S., Kim, J.S., Cho, H.S., Rha, D.S., Kim, J.M., Park, J.D., Choi, B.S., Lim, R., Chang, H.K., Chung, Y.H., Kwon, I.H., Jeong, J., Han, B.S. and Yu, I.J. (2008): Twenty-eight-day oral toxicity, genotoxicity, and gender-related tissue distribution of silver nanoparticles in Sprague-Dawley rats. Inhal. Toxicol., 20, 575-583.

Kim, Y.S., Song, M.Y., Park, J.D., Song, K.S., Ryu, H.R., Chung, Y.H., Chang, H.K., Lee, J.H., Oh, K.H., Kelman, B.J., Hwang, I.K., Yu, I.J. (2010): Subchronic oral toxicity of silver nanoparticles. Part. Fibre Toxicol., 7, 20.

Kokura, S., Handa, O., Takagi, T., Ishikawa, T., Naito, Y. and Yoshikawa, T. (2010): Silver nanoparticles as a safe preservative for use in cosmetics. Nanomedicine, 6, 570-574.

Korani, M., Rezayat, S.M., Gilani, K., Arbabi Bidgoli, S. and Adeli, S. (2011): Acute and subchronic dermal toxicity of nanosilver in guinea pig. Int. J. Nanomedicine, 6, 855-862.

Labouta, H.I. and Schneider, M. (2013): Interaction of inorganic nanoparticles with the skin barrier: current status and critical review. Nanomedicine, 9, 39-54.

Lankveld, D.P.K., Oomen, A.G., Krystek, P., Neigh, A., Troost-de Jong, A., Noorlander, C.W., Van Eijkeren, J.C.H., Geertsma,
R.E. and De Jong, W.H. (2010): The kinetics of the tissue distribution of silver nanoparticles of different sizes. Biomaterials, 31, 8350-8361.

Larese Filon, F., Mauro, M., Adami, G., Bovenzi, M., Crosera, M. (2015): Nanoparticles skin absorption: New aspects for a safety profile evaluation. Regul. Toxicol. Pharmacol., 72, 310-322.

Loeschner, K., Hadrup, N., Qvortrup, K., Larsen, A., Gao, X., Vogel, U., Mortensen, A., Lam, H.R. and Larsen, E.H. (2011): Distribution of silver in rats following 28 days of repeated oral exposure to silver nanoparticles or silver acetate. Part Fibre Toxicol., 8, 18.

Lok, C.N., Ho, C.M., Chen, R., He, Q.Y., Yu, W.Y., Sun, H., Tam, P.K., Chiu, J.F. and Che, C.M. (2007): Silver nanoparticles: partial oxidation and antibacterial activities. J. Biol. Inorg. Chem., 12, 527-534.

Munger, M.A., Radwanski, P., Hadlock, G.C., Stoddard, G., Shaaban, A., Falconer, J., Grainger, D.W. and Deering-Rice, C.E. (2014): In vivo human time-exposure study of orally dosed commercial silver nanoparticles. Nanomedicine, 10, 1-9.

Ostrowski, A., Nordmeyer, D., Boreham, A., Brodwolf, R., Mundhenk, L., Fluhr, J.W., Lademann, J., Graf, C., Ruhl, E., Alexiev, U. and Gruber, A.D. (2014): Skin barrier disruptions in tape stripped and allergic dermatitis models have no effect on dermal penetration and systemic distribution of AHAPS-functionalized silica nanoparticles. Nanomedicine, 10, 1571-1581.

Patzelt, A. and Lademann, J. (2013): Drug delivery to hair follicles. Expert Opin. Drug Deliv., 10, 787-797.

Rigo, C., Ferroni, L., Tocco, I., Roman, M., Munivrana, I., Gardin, C., Cairns, W.R., Vindigni, V., Azzena, B., Barbante, C., Zavan, B. (2013): Active silver nanoparticles for wound healing. Int. J. Mol. Sci., 14, 4817-4840.

Samberg, M.E., Oldenburg, S.J. and Monteiro-Riviere, N.A. (2010): Evaluation of silver nanoparticle toxicity in skin in vivo and keratinocytes in vitro. Environ. Health Perspect., 118, 407-413.

Stefaniak, A.B., Duling, M.G., Lawrence, R.B., Thomas, T.A., LeBouf, R.F., Wade, E.E., Virji, M.A. (2014): Dermal exposure potential from textiles that contain silver nanoparticles. Int. J. Occup. Environ. Health, 20, 220-234.

Tang, J., Xiong, L., Wang, S., Wang, J., Liu, L., Li, J., Yuan, F. and Xi, T. (2009): Distribution, translocation and accumulation of silver nanoparticles in rats. J. Nanosci. Nanotech., 9, 4924-4932.

Treinen-Moslen, M. (2001) Toxic responses of the liver. In: Klaassen CD (ed.) Casarett and Doull's Toxicology, The basic science of poisons McGraw-Hill, USA, pp.471-490. 